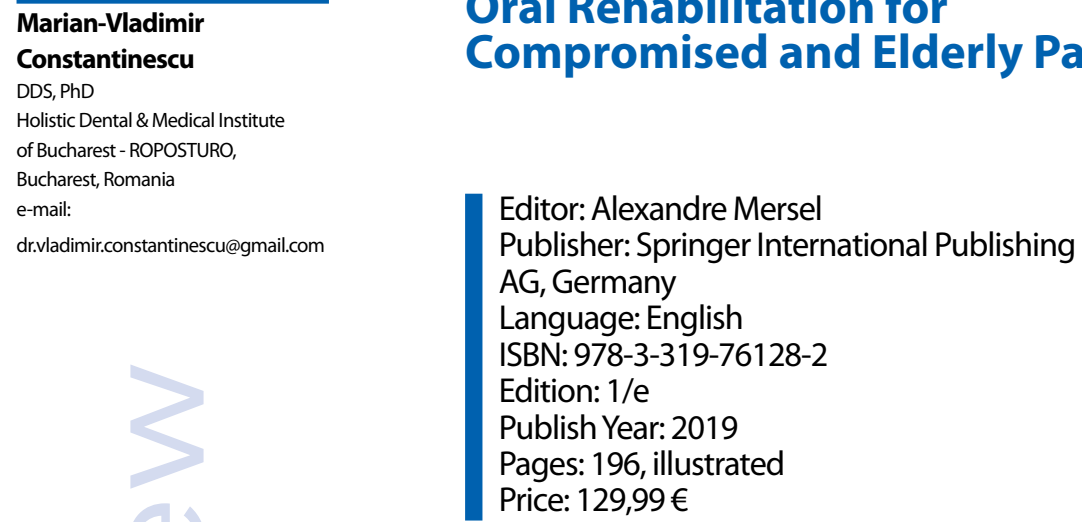

Editor: Alexandre Mersel

Publisher: Springer International Publishing

AG, Germany

Language: English

ISBN: 978-3-319-76128-2

Edition: $1 / \mathrm{e}$

Publish Year: 2019

Pages: 196, illustrated

Price: $129,99 €$

\section{Oral Rehabilitation for Compromised and Elderly Patients}

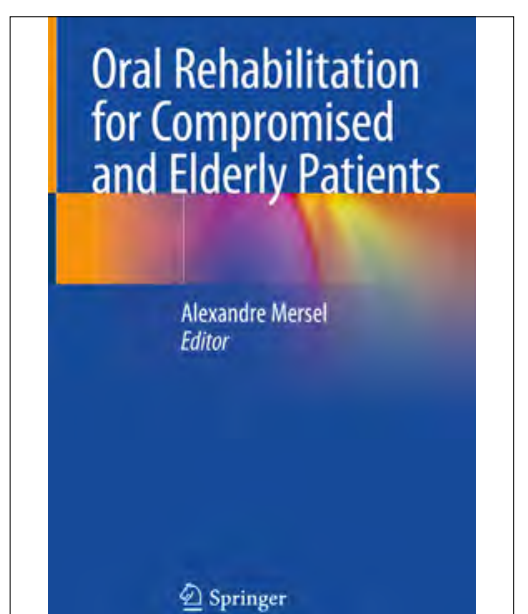

The World Health Organization (WHO) estimates that life expectancy will increase in the next 30 years and the cohort of elderly people will have an important impact on daily dental practice.

Professor Alexandre Mersel, renowned authority in geriatric dentistry, together with his 11 co-workers have drafted the book "Oral Rehabilitation for Compromised and Elderly Patients" to teach dentists how they can better provide evidence-based dental treatment for their aging patients.

This text is divided in 11 chapters. The first chapter talks about demography and aging. The correlation between systemic diseases and oral health is presented in chapter two. The third chapter explains treatment approaches for periodontal diseases and the modifiers which are linked to the biological changes due to age, chronic diseases, medications and socio-economical issues. An entire chapter provides a description of the physiological changes in endodontics, and how to decide and process a root canal treatment in elder patients. Chapter five presents some general concepts of TMD etiology, diagnosis and management. The following chapter speaks about the masticatory functions and nutritional status - considerations for an ageing population. Chapter seven and eight are dedicated to the caries destruction of the remaining teeth in the elder patients and minimalistic approach for conservative restorations such as the Minimal Invasive Treatment (MIT), infiltrations and the use of mesenchymal stem cells (MSCs) which may represent an autologous source of cells for tooth regenerative research. Chapter nine makes an evaluation of transitional or immediate complete dentures and should be specific because it has to cope with changes in the oral conditions initiated by ageing; and the last two chapters underline the limits of complete denture rehabilitation and the uniqueness of implant overdentures.

Because of the huge increase in the elderly population the general practitioner in dentistry is faced with important issues and this book with simple and easy procedures will help him overcome difficulties in these areas. Each general practitioner in dentistry who reads the book by Professor Alexandre Mersel and his renowned co-workers will find explanatory information on how to handle complex oral rehabilitation for compromised and elderly patients. 